\title{
ON THE MINIMALITY OF TAME MODELS IN THE ISOLS
}

\author{
JOSEPH BARBACK
}

(Communicated by Andreas R. Blass)

\begin{abstract}
Based on the work of Hirschfeld, it is known that there is a close connection between models for the $\Pi_{2}^{0}$ fragment of arithmetic and homomorphic images of the semiring of recursive functions. This fragment of arithmetic includes most of the familiar results of classical number theory. There is a realization of this fragment in the isols in systems called tame models. In this paper a new proof is given to the following result of Ellentuck and McLaughlin on the minimality of tame models: If two tame models share an infinite element, then the models are equal.
\end{abstract}

\section{INTRODUCTION}

We will assume that the reader is familiar with topics in the theory of isols. Our paper is concerned with a particular algebraic system of isols called a tame model and with a minimality result about tame models. The result was discovered by Ellentuck and McLaughlin independently. It states that if $\operatorname{Tm}(A)$ and $\operatorname{Tm}(B)$ are tame models that share an infinite element, then $\operatorname{Tm}(A)=\operatorname{Tm}(B)$. It may be obtained as a consequence of Theorems 3.2(1), 3.5, and 3.10 of [10] and the fact that the intersection of any two tame models is a model of all true $\Pi_{2}^{0}$ statements of arithmetic, a fact that can be deduced from Lemma 2 of [9]. The aim of our paper is to give another proof to the result.

Tame models were introduced in [2]. Let $\mathscr{U}$ be the collection of all increasing recursive functions of one variable. If $f$ is any recursive function, then $f_{\Lambda}$ will denote the extension of $f$ to the isols. If $A$ is a regressive isol and $f$ is an increasing recursive function, then the value of $f_{\Lambda}(A)$ is also a regressive isol. To each regressive isol $A$, let $\operatorname{Tm}(A)$ be the collection of isols defined by

$$
\operatorname{Tm}(A)=\left(f_{\Lambda}(A): f \in \mathscr{U}\right) .
$$

If $A$ is a finite isol, then $\operatorname{Tm}(A)=\omega$. The interesting setting for the collections $\operatorname{Tm}(A)$ is when $A$ is an infinite regressive isol, for then $\operatorname{Tm}(A)$ contains $\omega$ together with infinitely many infinite isols. It is always true that $\operatorname{Tm}(A)$ is a countable collection, and it is closed under addition and multiplication. Some of the elements in $\operatorname{Tm}(A)$ are $A, A^{2}$, and $2^{A}$.

Received by the editors December 9, 1991 and, in revised form, March 18, 1992.

1991 Mathematics Subject Classification. Primary 03D50. 


\section{TAME MODELS}

Let $A$ be an infinite regressive isol. The algebraic system $[\operatorname{Tm}(A),+, \cdot]$ has embedded within it the familiar system $[\omega,+, \cdot]$; we shall simply write $\operatorname{Tm}(A)$ and $\omega$ for these systems. It will depend upon the choice of $A$ as to how close one may find familiar arithmetic properties among the isols of $\operatorname{Tm}(A)$. For example, if $A$ is neither even nor odd, then not all elements of $\operatorname{Tm}(A)$ have parity.

Tame models were introduced in an effort to find algebraic systems in the isols with arithmetic properties that may more closely resemble those in the familiar arithmetic of $\omega$. Based upon an earlier notion of a torre isol, Ellentuck introduced in [5] the concept of a recursively strongly torre isol. An infinite regressive isol $A$ is called recursively strongly torre if either $f_{\Lambda}(A) \leq g_{\Lambda}(A)$ or $g_{\Lambda}(A) \leq f_{\Lambda}(A)$, for all increasing recursive functions $f$ and $g$. In an earlier work [12] Nerode showed the existence of regressive isols that are recursively strongly torre. Proved in [2] is the existence of infinite regressive isols $A$ that are recursively strongly torre, and with the additional property that if $f$ and $g$ are any increasing recursive functions with $f_{\Lambda}(A) \leq g_{\Lambda}(A)$, then $f_{\Lambda}(A)+$ $h_{\Lambda}(A)=g_{\Lambda}(A)$ for some increasing recursive function $h$. When $A$ is an infinite regressive isol with these two properties, then $\operatorname{Tm}(A)$ is called a tame model.

Let $\operatorname{Tm}(C)$ be a tame model. In [2] it was shown that the following properties are true:

(a) All elements of $\operatorname{Tm}(C)$ have parity, and all elements are comparable by the relation $\leq$ among isols.

(b) If $r$ is any recursive function of $n$-variables and $U_{1}, \ldots, U_{n}$ are members of $\operatorname{Tm}(C)$, then $r_{\Lambda}\left(U_{1}, \ldots, U_{n}\right)$ is also a member of $\operatorname{Tm}(C)$.

(c) If $U$ is a member of $\operatorname{Tm}(C)$ and $U$ is a prime isol, then $U$ may be expressed in the following way: $U=\operatorname{pr}_{\Lambda}(A)$ where $\mathrm{pr}$ is the principal function for the set of prime numbers and $A$ is an element of $\operatorname{Tm}(C)$.

(d) If $U$ and $V$ belong to $\operatorname{Tm}(C)$ and $U \leq^{*} V$, then $U \leq V$.

An important result about the arithmetic properties of tame models was proved by McLaughlin in [8]. It establishes that every tame model is a model for the true $A E$ sentences of Peano Arithmetic. We shall now begin the steps that lead us to the minimality property of tame models.

Definition. Let $\operatorname{Tm}(E)$ be a tame model and $B$ an element of $\operatorname{Tm}(E)$. Let $\operatorname{Pre}(B, \operatorname{Tm}(E))=(S \in \operatorname{Tm}(E): S \leq B)$.

Lemma L1. Let $\operatorname{Tm}(E)$ and $\operatorname{Tm}(F)$ be tame models. Let $B$ be an isol in both $\operatorname{Tm}(E)$ and $\operatorname{Tm}(F)$. Then $\operatorname{Pre}(B, \operatorname{Tm}(E))=\operatorname{Pre}(B, \operatorname{Tm}(F))$.

Proof. We shall just verify the one inclusion that $\operatorname{Pre}(B, \operatorname{Tm}(E))$ is a subset of $\operatorname{Pre}(B, \operatorname{Tm}(F))$, as the other inclusion may be shown in a similar way. If $B$ is a finite isol, then the desired conclusion is easy, as then both $\operatorname{Pre}(B, \operatorname{Tm}(E))$ and $\operatorname{Pre}(B, \operatorname{Tm}(F))$ consist of the values $0, \ldots, B$.

Let us assume now that $B$ is infinite. Then $\omega$ is a subset of both $\operatorname{Pre}(B, \operatorname{Tm}(E))$ and $\operatorname{Pre}(B, \operatorname{Tm}(F))$. Let $Y$ be in $\operatorname{Pre}(B, \operatorname{Tm}(E))$. To show that $Y$ is also in $\operatorname{Pre}(B, \operatorname{Tm}(F))$, we may assume that $Y$ is infinite. 
Let $g$ and $h$ be increasing recursive functions such that

$$
g_{\Lambda}(E)=h_{\Lambda}(F)=B \text {. }
$$

Because $\operatorname{Tm}(E)$ is a tame model and $Y \in \operatorname{Pre}(B, \operatorname{Tm}(E))$, there will then also be increasing recursive functions $p$ and $r$ with

$$
Y=p_{\Lambda}(E) \text { and } p_{\Lambda}(E)+r_{\Lambda}(E)=g_{\Lambda}(E) .
$$

The functions $g$ and $p$ range over infinite sets, since $B$ and $Y$ are infinite isols. Let the function $u$ be defined by

$$
u(m)=(\mu y)(g(y) \geq m \text { and } p(y)+r(y) \geq m)
$$

for $m \in \omega$. Then $u$ is everywhere defined, since the functions $g, p$, and $r$ are increasing, and the functions $g$ and $p$ have infinite ranges. By its definition, it is easy to see that $u$ is an increasing recursive function. Hence $u \in \mathscr{U}$.

The following two properties about the functions defined above are true. (We shall write $\circ$ for the composition operation between functions.) For all numbers $e, e_{0}$, and $f$,

$$
\begin{gathered}
\text { if } g(e)=h(f), \text { then }(u \circ g)(e)=(u \circ h)(f), \\
\text { if } e_{0}=(u \circ g)(e) \text { and } p(e)+r(e)=g(e) \text {, then } p\left(e_{0}\right)=p(e) .
\end{gathered}
$$

Property (3) is clear. To verify (4), assume that $e_{0}=(u \circ g)(e)$ and $p(e)+r(e)=$ $g(e)$. Then substitution in the definition of $u$ gives

$$
e_{0}=(\mu y)(g(y) \geq g(e) \text { and } p(y)+r(y) \geq p(e)+r(e)) .
$$

Hence, $e_{0} \leq e$ and $p\left(e_{0}\right)+r\left(e_{0}\right) \geq p(e)+r(e)$. Also, because the functions $p$ and $r$ are increasing, $e_{0} \leq e$ implies $p\left(e_{0}\right)+r\left(e_{0}\right) \leq p(e)+r(e)$. Therefore, $p\left(e_{0}\right)+r\left(e_{0}\right)=p(e)+r(e)$. Then $p\left(e_{0}\right)=p(e)$, since both $p$ and $r$ are increasing functions. Hence, property (4) is also true.

Each of the statements (3) and (4) corresponds to a Horn sentence that is true in $\omega$. If we now apply the metatheorem of Nerode, we can conclude that each of the statements has an extension to the isols that is also true. In view of (1) and the extension of (3) to the isols, one obtains $(u \circ g)_{\Lambda}(E)=(u \circ h)_{\Lambda}(F)$. Let $E_{0}=(u \circ g)_{\Lambda}(E)$. Then $E_{0}=(u \circ h)_{\Lambda}(F)$, and

$$
E_{0} \in \operatorname{Tm}(F),
$$

since $(u \circ h)$ is an increasing recursive function. By combining (2) with the extension of (4) to the isols, we get

$$
Y=p_{\Lambda}(E)=p_{\Lambda}\left(E_{0}\right) .
$$

Lastly, from (5), (6), and the fact that $p$ is an increasing recursive function, it follows that $Y \in \operatorname{Tm}(F)$. Therefore, $Y$ belongs to $\operatorname{Pre}(B, \operatorname{Tm}(F))$. This completes our proof.

Lemma L2. Let $\operatorname{Tm}(E)$ be a tame model. Let $U$ be an element of $\operatorname{Tm}(E)$ with $U$ infinite. There is then an increasing recursive function $r$, with $E \leq r_{\Lambda}(U)$.

Proof. Let $U=u_{\Lambda}(E)$ with $u$ an increasing recursive function. Define the function $r$ by $r(n)=(\mu y)(u(y)>n)$ for each number $n$. Then $u$ has an infinite range, since $U$ is an infinite isol. Also, $e<r(u(e))$ for all numbers $e$, 
since $u$ is increasing and has an infinite range. Hence,

$$
e \leq r(u(e))
$$

for all numbers $e$. Statement (7) may be extended to the isols; and since $E$ is a regressive isol, we may then obtain $E \leq^{*} r_{\Lambda}\left(u_{\Lambda}(E)\right)$. Therefore, $E \leq r_{\Lambda}\left(u_{\Lambda}(E)\right)$ by property $(\mathrm{d})$ and the fact that $\operatorname{Tm}(E)$ is a tame model. Hence, $E \leq r_{\Lambda}(U)$, and the desired result follows.

Lemma L3. Let $\operatorname{Tm}(E)$ be a tame model and $U$ an infinite element of $\operatorname{Tm}(E)$. Let $V$ be any element of $\operatorname{Tm}(E)$. Then $V \leq g_{\Lambda}(U)$ for an increasing recursive function $g$.

Proof. $V=v_{\Lambda}(E)$ with $v$ an increasing recursive function. By Lemma L2 $E \leq$ $r_{\Lambda}(U)$ for an increasing recursive function $r$. Therefore, $v_{\Lambda}(E) \leq v_{\Lambda}\left(r_{\Lambda}(U)\right)$, since $v$ is increasing recursive, $\operatorname{Tm}(E)$ is a tame model, and property (d). Setting $g=(v \circ r)$ gives $g$ an increasing recursive function and $V \leq g_{\Lambda}(U)$.

Theorem T1 (Ellentuck and McLaughlin). Let $\operatorname{Tm}(A)$ and $\operatorname{Tm}(B)$ be tame models. Let $U$ be an infinite element of $\operatorname{Tm}(A)$ and $\operatorname{Tm}(B)$. Then $\operatorname{Tm}(A)=$ $\operatorname{Tm}(B)$.

Proof. We shall simply verify that $\operatorname{Tm}(A)$ is a subset of $\operatorname{Tm}(B)$, as the other inclusion may be similarly shown. Let $V$ be an element of $\operatorname{Tm}(A)$. By Lemma L3 there is an increasing recursive function $g$, with $V \leq g_{\Lambda}(U)$. Then $g_{\Lambda}(U)$ is an element of both $\operatorname{Tm}(A)$ and $\operatorname{Tm}(B)$.

By Lemma L1 it follows that $\operatorname{Pre}\left(g_{\Lambda}(U), \operatorname{Tm}(A)\right)=\operatorname{Pre}\left(g_{\Lambda}(U), \operatorname{Tm}(B)\right)$. Since $V$ is an element of the first set, it follows that $V$ will be an element of $\operatorname{Tm}(B)$. Hence, $\operatorname{Tm}(A)$ is a subset of $\operatorname{Tm}(B)$, and this completes our proof.

Since every tame model contains $\omega$, we may obtain directly the following corollary to Theorem T1.

Corollary C1. Let $\operatorname{Tm}(A)$ and $\operatorname{Tm}(B)$ be tame models. Then either $\operatorname{Tm}(A) \cap$ $\operatorname{Tm}(B)=\omega$ or $\operatorname{Tm}(A)=\operatorname{Tm}(B)$.

\section{ElEMENTARY MODELS}

An infinite regressive isol $A$ is called recursively torre, if for all recursive sets $\alpha$, one has $A \in \alpha_{\Lambda}$ or $A \in(\omega-\alpha)_{\Lambda}$. Recursively torre isols are studied in [1] and [5]. In [1] it was shown that $A$ is recursively torre if $f_{\Lambda}(A) \leq^{*} g_{\Lambda}(A)$ or $g_{\Lambda}(A) \leq^{*} f_{\Lambda}(A)$ for all increasing recursive functions $f$ and $g$. In addition, if $A$ is recursively torre, then the system $\operatorname{Tm}(A)$ generates an integral domain in the isolic integers.

A system $\operatorname{Tm}(A)$, when $A$ is an infinite regressive isol that is recursively torre, will be called an elementary model. It is easy to see that a recursively strongly torre regressive isol is recursively torre, since among regressive isols $D \leq E$ implies $D \leq \leq^{*} E$. Therefore, each tame model is also an elementary model. In [8] McLaughlin proved that every elementary model is a model of the true universal sentences of Peano Arithmetic. Our interest here in elementary models is to express the fact that it is open at the moment whether elementary models are also minimal, as are the tame models. 


\section{ACKNOWLEDGMENT}

I would like to express my appreciation to T. G. McLaughlin for pointing out that the main result of the paper may be obtained as a consequence of his work in [10] and to the referee for the suggestion to include the additional reference [9]. I also express my appreciation to the referee for other suggestions to correct and improve the paper.

\section{REFERENCES}

1. J. Barback, On tame regressive isols, Houston J. Math. 8 (1982), 153-159.

2. __ Tame models in the isols, Houston J. Math. 12 (1986), 163-175.

3. _ Corrections for two papers on tame regressive isols and tame models in the isols, Houston J. Math. 13 (1987), 301-302.

4. J. C. E. Dekker and J. Myhill, Recursive equivalence types., Univ. of California Publ. Math. (N.S.) 3 (1960), 67-214.

5. E. Ellentuck, Diagonal methods in the theory of isols, Z. Math. Logik Grundlag. Math. 26 (1980), 193-204.

6. J. Hirschfeld, Models of arithmetic and recursive functions, Israel J. Math. 20 (1975), 111126.

7. T. G. McLaughlin, Regressive sets and the theory of isols, Lecture Notes in Pure and Appl. Math., vol. 66, Dekker, New York, 1982.

8. $\ldots$, Nerode semirings and Barback's 'Tame Models', Houston J. Math. 12 (1986), 211223.

9. __ Some properties of AE models in the isols, Proc. Amer. Math. Soc. 97 (1986), 495502.

10. _ـ Sub-arithmetical ultrapowers: a survey, Ann. Pure Appl. Logic 49 (1990), 143-191.

11. A. Nerode, Extensions to isols, Ann. of Math. (2) 73 (1961), 362-403.

12. _ Diophantine correct non-standard models in the isols, Ann. of Math. (2) 84 (1966), 421-432.

Department of Mathematics, Buffalo State College, Buffalo, New York 14222-1095 